\title{
Social Network-Proximity Association: Preliminary Evaluation of Giraffe Sociality in a Zoo-Housed Group
}

\author{
Chiara Grasso", Giorgia Poso ${ }^{2}$, and Christian Lenzi ${ }^{1, *}$ \\ ${ }^{1}$ Associazione ETICOSCIENZA, Turin, Italy \\ ${ }^{2}$ Faculty of Veterinary Medicine, University of Teramo, Italy \\ *Corresponding author (Email: lenzichristian@hotmail.it)
}

Citation - Grasso, C., Poso, G., \& Lenzi, C. (2022). Social network-proximity association: Preliminary evaluation of giraffe sociality in a zoo-housed group. Animal Behavior and Cognition, 9(1), 80-88. https://doi.org/10.26451/abc.09.01.07.2022

\begin{abstract}
Giraffes (Giraffa camelopardalis) are found in zoos all over the world. In recent years, numerous researchers have documented complex sociality in these mammals. They highlighted that giraffes have non-random preferences in their choices of social partners, which can depend on various factors such as age, sex, and kinship. One of the still little-known aspects is how the social structure of giraffes is formed in captivity. Moreover, the scientific literature about some aspects of the social structure of giraffes in captivity (i.e., proximity or affiliative interactions) is scarce. Our hypothesis was that there would be an association between the social network, based on affiliative reciprocal interactions, and physical proximity within a group of six giraffes ( 5 females and 1 male) living in a zoo. To test this hypothesis, in addition to the ethological observations, we also used a Geographical Information System (GIS) to study the position of the individuals within the daytime installation. Most of the giraffes had a high number of mutual dyadic interactions, which is connected to high group cohesion. Also, each individual actively selected social partners and formed non-random social bonds. Nevertheless, our hypothesis that there would be a social network-physical proximity association, was confirmed for one dyad and partially confirmed for the other two. The results of this study can be useful to increase the knowledge of giraffe sociality and to develop GIS as a new application in zoo studies.
\end{abstract}

Keywords - Affiliative, Giraffe, GIS, Proximity, Sociality

Giraffes (Giraffa camelopardalis) are found in zoos all over the world and many studies have analyzed their social behavior in captivity (e.g., Guarino et al., 2002). These mammals have a fission-fusion social structure characterized by the union and division of its component units. Furthermore, their social hierarchy is closely linked to the sex and age of individuals, with males and old females as leaders of the group (Bashaw et al., 2007; Horovà et al., 2015).

Giraffes establish long-lasting social bonds based on many factors, such as kinship and sex (Perry, 2011; Skinner \& Mitchell, 2011). Moreover, as documented both in the wild and in captivity, giraffes have a non-random social preference for conspecifics (Bashaw et al., 2007; Lewton \& Rose, 2019; Malyjurkova et al., 2014).

A relevant aspect of relationship is proximity. A study conducted in the wild by VanderWaal et al. (2014) showed that each individual's space use was related to the social interactions they established. In captivity, physical distance between individuals and their affiliative behaviors are not randomly distributed (Bashaw et al., 2007; Garry, 2012; Perry, 2011). 
There are multiple tools that allow us to investigate social proximity. Geographical Information System (GIS) seems to be very useful both in the wild and in captive environments (Guarino et al., 2002 Swetnam \& Reyers, 2011). This tool has been used to study various aspects of giraffe behavior in the wild (Brand, 2007; Nogueira, 2015), but there is no record of its use in captive groups.

The aim of this research is to study the relation between group social networks and physical proximity, using GIS methodology, in a zoo-housed group of giraffes.

\section{Method}

\section{Study Site and Subjects}

Six giraffes were observed from March to May 2017 at Bioparc Valencia, Spain: 5 females and 1 male (Table 1). The giraffes were in a daytime installation with an available area of about $900 \mathrm{~m}^{2}$ (Figure 1). The study period had been preceded by ad libitum sessions (Altmann, 1974). During the observations, Zora (I3, one of the females) was 14-months pregnant and the male Julius (I1) was neutered.

\section{Figure 1}

Daylight Giraffe Enclosure

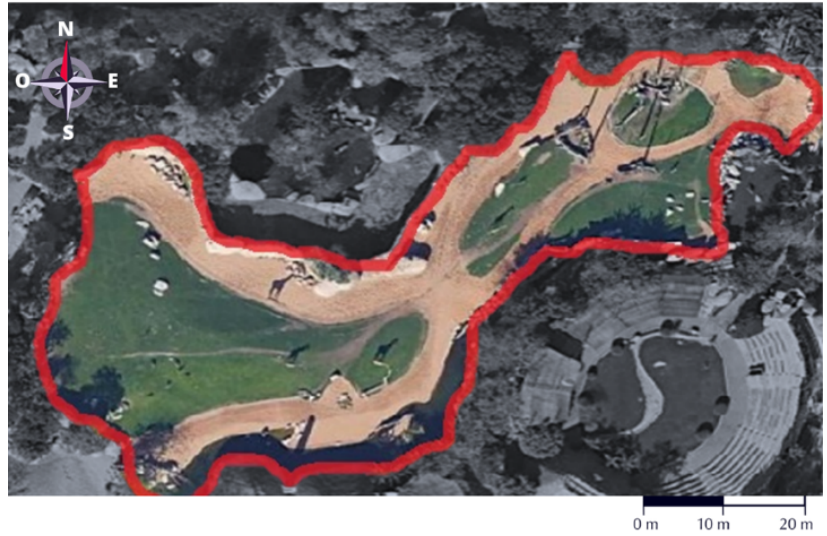

Note. The study area is highlighted with a red line.

\section{Table 1}

General Information Related to the Observed Animals

\begin{tabular}{|c|c|c|c|c|c|c|}
\hline Name & Code & Born & Sex & Arrived & Mother & Father \\
\hline Julius & I1 & $30 / 11 / 2005$ & Male & 03/08/2007 & unknown & unknown \\
\hline Ché & $\mathrm{I} 2$ & $5 / 11 / 1995$ & Female & $04 / 08 / 2007$ & unknown & unknown \\
\hline Zora & $\mathrm{I} 3$ & $26 / 01 / 2006$ & Female & $13 / 04 / 2007$ & unknown & unknown \\
\hline Africa & I4 & 05/07/2011 & Female & birth date & Ché & Julius \\
\hline Sahira & I5 & $11 / 05 / 2014$ & Female & birth date & Zora & Julius \\
\hline Lluna & I6 & $29 / 12 / 2015$ & Female & birth date & Zora & Julius \\
\hline
\end{tabular}


The giraffes shared the zoo exhibit with: 10 Thomson's gazelles (Eudorcas thomsonii), 10 impalas (Aepyceros melampus), 4 blesboks (Damaliscus pygargus phillipsi), 3 waterbucks (Kobus ellipsiprymnus), 2 jabiru storks (Jabiru mycteria), 20 white Australian ibis (Threskiornis molucca), and other waterfowl (Anseriformes).

\section{Affiliative Behaviors and GIS Proximity Analysis}

Data were collected by a single observer, equipped with $8 \times 21126 \mathrm{~mm} / 1000 \mathrm{~m}$ binoculars. Before the observation period, the observer followed a one-month training period using video records and live sessions. The selected observation location (zoo cafeteria) was about $3 \mathrm{~m}$ away from the enclosure.

Behavioral observations were conducted using both focal and instantaneous scan samplings (Altmann, 1974), performed simultaneously from 10:00 to 19:00 h for three months. All subjects were the target of ethological records for equal amounts of time. We avoided observing during feeding times when all giraffes might be in close proximity for non-social reasons. The zookeepers distributed the food in different areas of the exhibit, without a standardized protocol. It was therefore not possible to establish observations during feeding behavior, although previous studies (in captivity, Bashaw et al., 2007, and in the wild, Prehn et al., 2019) showed important implications for the social structure of the group.

Each focal observation lasted $10 \mathrm{~min}$ (with a 20 min break from each other), with 18 sessions per day, resulting in a total of 1171 focal samplings. For the aims of this study, we selected three affiliative behaviors (Table 2). The social interactions within the group were studied through the analysis of dyads. These dyads can display unidirectional (i.e., only one of the two individuals is the actor and the other is the receiver) or bidirectional (i.e., two individuals participate as actors and recipients) interactions. If two-way affiliative interactions are made at the same frequency, they were considered as reciprocated.

Data were analyzed using matrices, derived from the behavior frequency for each individual, that were processed by the software Socprog 2.4. We selected this software because it is designed to provide flexible analyses of social structure using data on interactions of identified individuals (Whitehead, 2009). A weighted adjacency matrix was built by bidirectional interactions.

We built a weighted and directed network sociogram (Makagon et al., 2012, Rose \& Croft, 2015), analyzing the social network of the giraffe group. Each node corresponds to an individual and interaction strengths ('high,' 'medium' and 'low') are represented by the thickness of the edge. Direction of the interactions is mapped on using arrows, which point away from the actor and toward the receiver. Relations strengths were analyzed by out-degrees and in-degrees. The interaction rate was calculated directly by the software, highlighting the differences in the levels of association between individuals.

At the end of the observation period, we obtained a total of 307 scan samplings ( 20 min interval between each scan). The position of each giraffe was drawn on a paper map (scale of 1:1000), indicated with dots and the identification number. Because we wanted to study the physical proximity, we used two neck-lengths apart as reference method (Bashaw et al., 2007). In particular, we drew a line as a junction between the points identifying the location. To facilitate the interpretation of the results, we used three categories: conjunction line and a half vertical bar (short distance), whole line with no bars in the middle (medium distance), and no line of conjunction (long distance). Moreover, the position and the behavior of each individual were georeferenced along with general and ethological information (e.g., observed animal's ID, time, date and behavior). To digitize the positions from the paper map, we used a digitizing tablet in a GIS environment (QGIS v. 3.4). Then, we applied a Kruskal-Wallis test by ranks followed by a post-hoc pairwise Mann Whitney test to compare median daily distances between pairs of individuals over the entire observation period. 
Table 2

Behavioral and Social Proximity Categories Used in the Study

\begin{tabular}{lll}
\hline Behavior & Code & Definition \\
\hline Nuzzling & $\mathrm{Sn}$ & $\begin{array}{l}\text { A tactile encounter by a giraffe's nose or muzzle to another giraffe's nose or any } \\
\text { other part of the body (anogenital area excluded) }\end{array}$ \\
$\begin{array}{ll}\text { Grooming } \\
\text { Rubbing }\end{array}$ & $\mathrm{Sg}$ & One giraffe grooms, licks, or bites another giraffe \\
\hline Social proximity & $\mathrm{Sr}$ & $\begin{array}{l}\text { One giraffe rubs its head or neck against another giraffe's body, sometimes leading } \\
\text { to an entwining of the necks }\end{array}$ \\
\hline $\begin{array}{l}\text { Short } \\
\text { Middle }\end{array}$ & $\mathrm{S}$ & $\begin{array}{l}\text { The observed individuals were close to each other, at such a distance that they could } \\
\text { have been touched only by lengthening the neck }(\mathrm{x} \leq 2 \mathrm{~m})\end{array}$ \\
& $\mathrm{M}$ & The animals were at a hypothetical distance of two bodies $(2<\mathrm{x} \geq 5 \mathrm{~m})$ \\
Long & $\mathrm{L}$ & The individuals were at a distance of more than two bodies $(\mathrm{x}>5 \mathrm{~m})$ \\
\hline
\end{tabular}

Note. The ethological definitions have been adapted from Bashaw et al. (2007), Seeber et al. (2012), and Ziarnowski \& Fenrich (2016).

\section{Results}

The network of affiliative interactions between dyads is shown in Figure 2. We found a double directionality of actions in which all subjects have both input and output arrows. Within the sociogram based on affiliative behavior, we found some differences for the interaction rate (high $=52.00$, medium $=26.05$, and low $=$ $0.10)$.

Figure 2

\section{Sociogram Based on Affiliative Interactions}

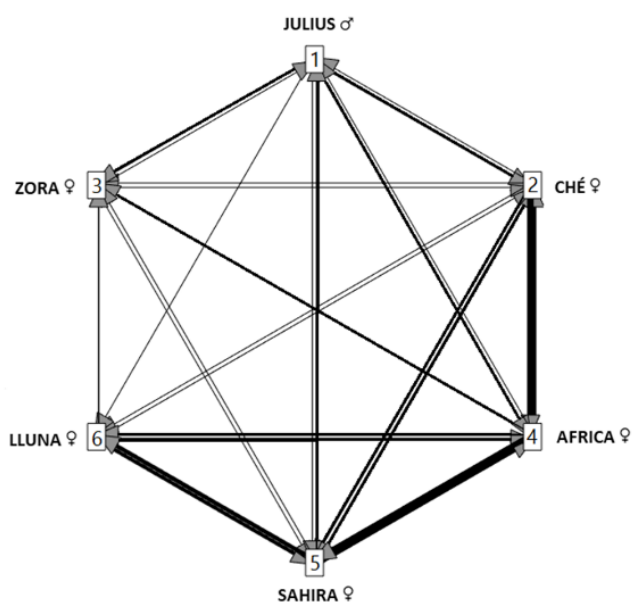

Note. Built using net draw. Wider arrows mean high frequency of affiliative behaviors, medium arrows mean medium frequency of affiliative behaviors and thin arrows means low frequency of affiliative behaviors. 
Most of the giraffes had a high number of mutual dyadic interactions. The group had a social profile with typical traits of giraffe social behavior, such as female cohesion and more solitary behavior of the male. The oldest female, Ché (I2), established five-way interactions, with four of them being reciprocated. Africa (I4) formed four bidirectional dyads; three of them were reciprocated. The frequencies of these three dyads stand out, since the two established with Ché (I2) and Sahira (I5) were high, and the one established with Lluna (I6) was medium. Sahira had two-way interactions with the entire group, with almost all dyads being reciprocated. Lluna and Zora (I3) had only two-way interactions with three group individuals, although all the dyads were reciprocated. Julius (I1) established bidirectional interactions with all the females except the youngest, Lluna. Furthermore, three of these dyads were reciprocated (I1-I3, I1-I4, and I1-I5).

Using GIS, we calculated the position of each individual within the enclosure and the interindividual physical distance. From georeferenced proximity analysis, the Kruskal-Wallis test showed statistically significant differences within median distances among individuals $(p<2.2 \mathrm{e}-16$, Figure 3$)$. Generally, for the male, Julius, there were no statistically significant differences within the distances between him and the other individuals. The median distance of the dyad Ché-Zora (2-3) was the highest, whereas the distances between Ché-Sahira (2-5) and Ché-Lluna (2-6) were shorter in comparison with the majority. Overall, the distance of Sahira-Lluna (5-6) was the shortest (Figure 3 and Table 3).

\section{Figure 3}

Kruskal-Wallis Test Analysis ( $\left.<2.2 e^{-16}\right)$ of Median Distances Among Individuals (Proximity)

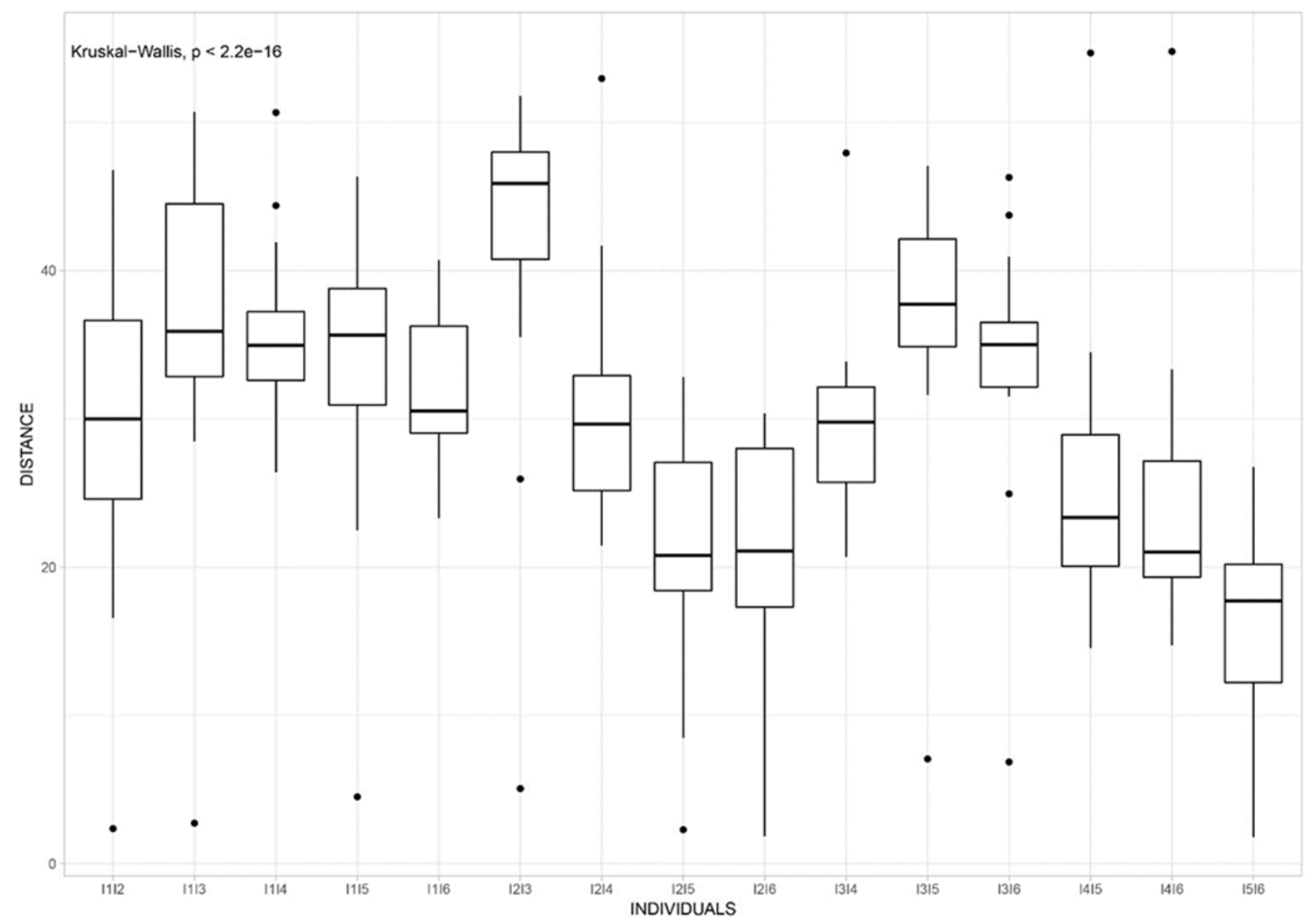

Note. Individuals: I1 (Julius), I2 (Ché), I3 (Zora), I4 (Africa), I5 (Sahira), and I6 (Lluna). 
Table 3

P-Values of the Post-Hoc Pairwise Mann Whitney Test

\begin{tabular}{|c|c|c|c|c|c|c|c|c|c|c|c|c|c|c|c|}
\hline & I1I2 & I1I3 & I1I4 & I1I5 & I1I6 & $\mathrm{I} 2 \mathrm{I} 3$ & I2I4 & I2I5 & I2I6 & I3I4 & I3I5 & I3I6 & I4I5 & I4I6 & I5I6 \\
\hline I1I 2 & - & - & - & - & - & - & - & - & - & - & - & - & - & - & - \\
\hline I1I3 & .374 & - & - & - & - & - & - & - & - & - & - & - & - & - & - \\
\hline I1I4 & .660 & 1 & - & - & - & - & - & - & - & - & - & - & - & - & - \\
\hline I1I5 & .950 & 1 & 1 & - & - & - & - & - & - & - & - & - & - & - & - \\
\hline I1I6 & 1 & .945 & .995 & 1 & - & - & - & - & - & - & - & - & - & - & - \\
\hline $\mathrm{I} 2 \mathrm{I} 3$ & .004 & .963 & .815 & .415 & .096 & - & - & - & - & - & - & - & - & - & - \\
\hline $\mathrm{I} 2 \mathrm{I} 4$ & 1 & .816 & .964 & 1 & 1 & .036 & - & - & - & - & - & - & - & - & - \\
\hline I2I5 & .344 & $<.001$ & $<.001$ & .002 & .038 & $<.001$ & .076 & - & - & - & - & - & - & - & - \\
\hline I2I6 & .227 & $<.001$ & $<001$ & .001 & .020 & $<.001$ & .041 & 1 & - & - & - & - & - & - & - \\
\hline I3I4 & 1 & .397 & .684 & .958 & 1 & .004 & 1 & .323 & .211 & - & - & - & - & - & - \\
\hline I3I5 & .367 & 1 & 1 & 1 & .942 & .965 & .811 & $<.001$ & $<.001$ & .389 & - & - & - & - & - \\
\hline I3I6 & .967 & .999 & 1 & 1 & 1 & .361 & 1 & .003 & .001 & .973 & .999 & - & - & - & - \\
\hline I $4 \mathrm{I} 5$ & .995 & .012 & .045 & .209 & .700 & $<001$ & .853 & .988 & .959 & .994 & .012 & .249 & - & - & - \\
\hline I4I6 & .911 & .002 & .008 & .056 & .346 & $<001$ & .519 & 1 & .999 & .898 & .002 & .070 & 1 & - & - \\
\hline I5I6 & .002 & $<001$ & $<001$ & $<001$ & $<001$ & $<001$ & $<001$ & .934 & .977 & .002 & $<001$ & $<001$ & .132 & .391 & - \\
\hline
\end{tabular}

Note. Statistically significant numerical values have been highlighted in bold type.

From the comparison of the social network data with the physical proximity results, our hypothesis was confirmed for the dyads Lluna-Sahira (I5-I6) and partially confirmed for Chè-Sahira (I2-I5) and ChèLluna (I2-I6).

\section{Discussion}

In the wild, giraffe populations have dramatically declined in abundance by almost $40 \%$ over the last 30 years, and the geographic ranges of the species have been significantly reduced or altered (O'Connor et al., 2019). The challenge now is to implement monitoring and surveillance of giraffes as a conservation priority (Deacon \& Tutchings, 2019). One of the key points concerning the understanding of this species and indirectly its preservation - is the social structure. As has been documented in previous studies, both in captivity and in the wild, this species exhibits complex inter-individual relations.

In agreement with other studies (Bashaw et al., 2007; Lewton \& Rose, 2019; Malyjurkova et al., 2014), our group of giraffes had non-random bonding preferences. As previously documented, factors such as age and parentage can influence the social structure of giraffes (Bercovitch \& Berry, 2013). Generally, we found high patterns of social affiliation within female dyads. All females had a high number of mutual dyadic interactions and, except Zora (I3, whose pregnancy likely affected her social interactions), showed reciprocated dyads with high or medium frequencies. These findings are consistent with those previously documented in wild herds (Bercovitch \& Berry, 2013; Carter et al., 2013; Shorrocks \& Croft, 2009) and in captivity (Lewton \& Rose, 2019).

Julius, the male (I1), appeared more solitary compared to the female individuals. Our results could be partially explained by the fact that male giraffes typically spend more time alone compared to the females (Bercovitch \& Berry, 2010; VanderWaal et al., 2014). Even if our male had more solitary behavior compared to the females, his mere presence could have modulated the social network within the group (Tarou et al., 2000). 
From the GIS analysis, it can be noted that Sahira and Lluna (I5-I6) tended to be closer to each other, and this relationship was confirmed by the analysis with Socprog. In addition, Ché-Lluna and ChéSahira (dyads I2-I6 and I2-I5) had high scores for physical proximity, though the ethological observations showed that the dyads were of low (I2-I6) and medium (I2-I5) frequencies.

Based on the results, it was assumed that only the youngest individuals (i.e., Lluna and Sahira, I6 and I5) had a close association between the affiliative behaviors and physical proximity. According to our observations, Zora's pregnancy (I3) during the study could have affected the relationship with her daughters, Lluna and Sahira (I6 and I5). The pregnant female may spend less time around her daughters than she normally would (Bercovitch \& Berry, 2013). Maybe for this reason, the two youngest had formed stable pairs with the elderly Ché (I2), as confirmed by affiliative-behavior observations and by GIS spatial analyses.

One of the potential limitations of our study is the fact that the group consisted of only six individuals, with a single male. However, it must be said that the social structure of this species is very dynamic (Bercovitch \& Berry, 2013). Thus, our group, with several social bonds based on kinship, can represent a good example for studying inter-individual relations.

An element that might affect how the social network structure was captured is the absence of observations during the feeding phase (due to a lack of standardization in the food distribution). This is a factor to consider, as previous studies (Bashaw et al., 2007, Prehn et al., 2019) documented non-random co-feeding social interactions.

Our results can be useful to better understand the social structure of giraffes, for all the various subspecies. Another interesting aspect regarding the application of our findings concerns the difference between groups in captivity and in the wild. Although in a zoo exhibit they cannot select the members within the social group, the study of giraffes in captivity can provide useful information for understanding their typical social structure (Bashaw et al., 2007).

According to our experience, GIS is a useful tool for studying social relations in zoos. The innovative application of GIS allowed us to investigate some aspects that were not possible to study only by ethological observations, e.g., social proximity. This method can be a valid tool for combining spatial information with ethological information. Our research, like others previously, showed how GIS can be effectively applied even in a limited space such as a zoo enclosure (Swetnam \& Reyers, 2011). In these kinds of studies, different issues should be considered, such as hand-mapping individuals and categorizing the distances, which potentially introduce opportunities for bias. In our research, we tried to minimize these influences. For example, the observer used various reference points present within the enclosure (such as trees, stones or streams) to precisely identify the spatial position of individuals. Furthermore, before the study, some test observations were carried out comparing the positions collected by the observer with the photographs taken above the exhibit. It would be interesting for other researchers to use and implement this methodology in the study of different aspects of giraffe sociality in captivity (for instance in combination with heat maps of ZooMonitor, Wark et al., 2019).

\section{Conclusion}

Non-random bonding was found in giraffes. A high number of mutual dyadic interactions were found, though not all were confirmed by GIS proximity analysis. However, our study showed that the group had typical features of giraffe social behavior such as female group cohesion and more male solitary behavior. An innovative aspect concerns the use of GIS. From our research, we documented how this methodology is a valuable tool with many applications that can be generalized to use with other zoo-housed species.

Conflict of Interest: The authors have no conflicts of interest to declare. 


\section{Acknowledgements}

We are very grateful to Federico Guillén-Salazar, Ester Orient Pérez, Mauro Fabrizio, Ludovico Frate, and Rossana Astolfi for their contributions, Ann Casper for the revision, and the Bioparc staff for the support.

\section{References}

Altmann, J. (1974). Observational study of behavior: Sampling methods. Behaviour, 49(3/4), 227-267.

Bashaw, M. J., Bloomsmith, M. A., Maple, T. L., \& Bercovitch, F. B. (2007). The structure of social relationships among captive female giraffe (Giraffa camelopardalis). Journal of Comparative Psychology, 121(1), 46-53.

Bercovitch, F. B., \& Berry, P. S. M. (2010). Ecological determinants of herd size in the Thornicroft's giraffe of Zambia. African Journal of Ecology, 48(4), 962-971.

Bercovitch, F. B., \& Berry, P. S. M. (2013). Herd composition, kinship and fission-fusion social dynamics among wild giraffe. African Journal of Ecology, 51(2), 206-216.

Brand, R. (2007). Evolutionary Ecology of Giraffes (Giraffa camelopardalis) in Etosha National Park, Namibia [unpublished doctoral dissertation]. Newcastle University.

Carter, K. D., Brand, R., Carter, J. K., Shorrocks, B., \& Goldizen, A. W. (2013). Social networks, long-term associations and age-related sociability of wild giraffes. Animal Behaviour, 86(5), 901-910.

Deacon, F., \& Tutchings, A. (2019). The South African giraffe Giraffa camelopardalis giraffa: A conservation success story. Oryx, 53(1), 45-48.

Garry, S. (2012). Analyses of captive behaviour and enclosure use in Rothschild giraffes (Giraffa camelopardalis rothschildi) housed at Paignton Zoo Environmental Park ${ }^{\circledR}$, The Plymouth Student Scientist, 5(2), 4-30.

Guarino, L., Jarvis, A., Hijmans, R. J., \& Maxted, N. (2002). Geographic information systems (GIS) and the conservation and use of plant genetic resources. In: Proceedings of the international conference on science and technology for managing plant genetic diversity in the 21st century (SAT21), Kuala Lumpur, Malaysia, 12-16 June 2000. International Plant Genetic Resources Institute, Rome, Italy.

Horovà, E., Brandlová, K., \& Gloneková, M. (2015). The first description of dominance hierarchy in captive giraffe: Not loose and egalitarian, but clear and linear, Plos One, 10(5), 1-13.

Lewton J., \& Rose, P. E. (2019). Evaluating the social structure of captive Rothschild's giraffes (Giraffa camelopardalis rothschildi): Relevance to animal management and animal welfare. Journal of Applied Animal Welfare Science, 23(2), 178-192.

Makagon, M. M., McCowan, B., \& Mench, J. A. (2012). How can social network analysis contribute to social behavior research in applied ethology? Applied Animal Behaviour Science, 138(3-4), 152-161.

Malyjurkova, L., Hejzlarova, M., Vymyslicka, P., \& Brandlova, K. (2014). Social preferences of translocated giraffes (Giraffa camelopardalis giraffa) in Senegal: Evidence for friendship among females? Agricultura Tropica et Subtropica, 47(1), 5-13.

Nogueira, J. (2015). Historical biogeography and climate change: Application of ecological niche-based models and GIS to the conservation of African elephants and giraffes [unpublished master's thesis]. University of Porto.

O'Connor, D., Stacy-Dawes, J., Muneza, A., Fennessy, J., Gobush, K., Chase, M. J., Brown, M. B., Bracis, C., Elkan, P., Zaberirou, A. R. M., Rabeil, T., Rubenstein, D., Becker, M. S., Phillips, S., Stabach, J. A., Leimgruber, P., Glikman, J. A., Ruppert, K., Masiaine, S., \& Mueller, T. (2019). Updated geographic range maps for giraffe, Giraffa spp., throughout sub-Saharan Africa, and implications of changing distributions for conservation. Mammal Review, 49(4), 285-299.

Perry, S. (2011). Social behaviour in captive reticulated giraffes (Giraffa camelopardalis reticulata): Analysis of enclosure use and social interactions between giraffes housed at Whipsnade. The Plymouth Student Scientist. $4(2), 50-65$.

Prehn, S. G., Laesser, B. E., Clausen, C. G., Jønck, K., Dabelsteen, T., \& Brask, J. B. (2019). Seasonal variation and stability across years in a social network of wild giraffe. Animal Behaviour, 157, 95-104.

Rose, P. E., \& Croft, D. P. (2015). The potential of social network analysis as a tool for the management of zoo animals. Animal Welfare, 24(2), 123-138.

Seeber, P. A., Ciofolo, I., \& Ganswindt, A. (2012). Behavioural inventory of the giraffe (Giraffa camelopardalis). NBC Research Notes, 5(1), 650 .

Shorrocks, B., \& Croft, D. P. (2009). Necks and networks: A preliminary study of population structure in the reticulated giraffe (Giraffa camelopardalis reticulata de Winston). African Journal of Ecology, 47(3), 374381. 
Skinner, J. D., \& Mitchell, G. (2011). Lung volumes in giraffes, Giraffa camelopardalis. Comparative Biochemistry and Physiology A, 158(1), 72-78.

Swetnam, R. D., \& B. Reyers (2011). Meeting the challenge of conserving Africa's biodiversity: The role of GIS, now and in the future. Landscape and Urban Planning, 100(4), 411-414.

Tarou, L. R., Bashaw, M. J. \& Maple, T. L. (2000). Social attachment in giraffe: Response to social separation. Zoo Biology, 19, 41-51.

VanderWaal, K. L., Wang, H., McCowan, B. Fushing, H., \& Isbell, L. A. (2014). Multilevel social organization and space use in reticulated giraffe (Giraffa camelopardalis). Behavioral Ecology, 25(1), 17-26.

Wark, J. D., Cronin, K. A., Niemann, T., Shender, M. A., Horrigan, A., Kao, A., \& Ross, M. R. (2019). Monitoring the behavior and habitat use of animals to enhance welfare using the ZooMonitor app. Animal Behavior and Cognition, 6, 158-167.

Whitehead, H. (2009). SOCPROG programs: Analysing animal social structures. Behavioural Ecology and Sociobiology, 63, 765-778.

Ziarnowski, P., \& Fenrich, K. (2016). Social behavior in a herd of captive male giraffes. The Pegasus Review: UCF Undergraduate Research Journal (URJ), 9(1), 29-39. 